\title{
Classifying Novel Phases of Spinor Atoms
}

\section{Citation}

Barnett, Ryan, Ari Turner, and Eugene Demler. 2006. “Classifying Novel Phases of Spinor

Atoms." Physical Review Letters 97 (18) (November 3). doi:10.1103/physrevlett.97.180412.

\section{Published Version}

doi:10.1103/PhysRevLett.97.180412

\section{Permanent link}

http://nrs.harvard.edu/urn-3:HUL.InstRepos:27893056

\section{Terms of Use}

This article was downloaded from Harvard University's DASH repository, and is made available under the terms and conditions applicable to Other Posted Material, as set forth at http:// nrs.harvard.edu/urn-3:HUL.InstRepos:dash.current.terms-of-use\#LAA

\section{Share Your Story}

The Harvard community has made this article openly available.

Please share how this access benefits you. Submit a story.

\section{Accessibility}




\title{
Classifying Novel Phases of Spinor Atoms
}

\author{
Ryan Barnett, ${ }^{1,2}$ Ari Turner, ${ }^{1}$ and Eugene Demler ${ }^{1}$ \\ ${ }^{1}$ Department of Physics, Harvard University, Cambridge, Massachusetts 02138, USA \\ ${ }^{2}$ Department of Physics, California Institute of Technology, MC 114-36, Pasadena, California 91125, USA
}

(Received 9 July 2006; published 3 November 2006)

\begin{abstract}
We consider many-body states of bosonic spinor atoms which, at the mean-field level, can be characterized by a single-particle wave function for the Bose-Einstein condensation and Mott insulating states. We describe and apply a classification scheme that makes explicit the spin symmetries of such states and enables one to naturally analyze their collective modes and topological excitations. Quite generally, the method allows classification of a spin $F$ system as a polyhedron with $2 F$ vertices. We apply the method to the many-body states of bosons with spins two and three. For spin-two atoms we find the ferromagnetic state, a continuum of nematic states, and a state having the symmetry of the point group of the regular tetrahedron. For spin-three atoms we obtain similar ferromagnetic and nematic phases as well as states having symmetries of various types of polyhedra with six vertices.
\end{abstract}

DOI: $10.1103 /$ PhysRevLett.97.180412

Ultracold atoms in either a single optical trap or in an optical lattice provide clean realizations of unique systems of spins which were previously studied only as toy mathematical problems (for a recent review, see [1]). Depending on which hyperfine state is populated, alkali atoms can have spin one or two. Different phases of spin-one alkali bosons have been experimentally realized [2-5] and considered theoretically [6-12]. Spin-two bosons have also been experimentally probed $[3,13,14]$ and theoretically studied for the case of a single optical trap $[15,16]$ as well as, very recently, an optical lattice [17,18]. Finally, the Stuttgart group has recently succeeded in obtaining a Bose-Einstein condensation of ${ }^{52} \mathrm{Cr}$ atoms [19] which are spin-three bosons. This was followed by theoretical work $[20,21]$ showing the possible types of phases that can be realized for such a spin-three system.

Classification schemes of single-particle states with nonzero spins are needed to describe both superfluid condensates and Mott insulating states of spinor bosonic atoms. However, such classification becomes increasingly more difficult for larger spins. For instance, classifying the state of a spin-half particle is straightforward; only knowledge of the expectation value of the spin operator $\langle\mathbf{F}\rangle$ is needed. On the other hand, for a spin-one particle, knowledge of the expectation value of the nematic tensor familiar from the classical theory of liquid crystals [22]

$$
Q_{a b}=\frac{1}{2}\left(F_{a} F_{b}+F_{b} F_{a}\right)-\frac{1}{3} \delta_{a b} F^{2}
$$

in addition to $\langle\mathbf{F}\rangle$ is required. Proceeding along these lines, one finds that for larger spin such a classification scheme becomes quite cumbersome since one needs to consider order parameters that involve higher-order products of spin operators, and a physical interpretation is not immediate.

In this Letter, we present an alternative classification scheme which will work well for large spin. This scheme allows the symmetries of a general spin $F$ particle to be represented by a polyhedron with $2 F$ vertices. To illustrate
PACS numbers: 05.30.Jp, 03.75.Hh, 03.75.Kk, 03.75.Mn

the method, we use it to discuss spin-two bosons which are naturally realized as a hyperfine state of alkali atoms. The previously discussed superfluid ferromagnetic, polar, and cyclic condensates $[15,16]$ are shown to have the transformation properties of a ferromagnet, a nematic (either uniaxial or biaxial), and a tetrahedron. In addition, we show that the nematic phase has an additional degeneracy at the mean-field level. We then show that states having similar transformation properties will be obtained in the Mott insulating state. Next, we show that the classification scheme immediately gives the number of Goldstone modes and allows one to classify topological excitations. In fact, in the biaxial nematic phase and the tetrahedratic phase, the topological excitations combine according to non-Abelian groups [23]. Finally, due to recent experimental interest, we will also discuss classification of spin-three bosons.

We will now outline our general classification scheme for a single spin. The key ingredient of our classification scheme is identifying states of spin $F$ particles with $2 F$ points on the unit sphere [24]. Consider a particle of spin $F$ in the state given by $|\psi\rangle=\sum_{\alpha=-F}^{F} A_{\alpha}|\alpha\rangle$, where $F_{z}|\alpha\rangle=$ $\alpha|\alpha\rangle$ and $A_{\alpha}$ are a set of normalized complex coefficients. To gain a physical understanding of this state, the idea is to find the set of "maximally polarized" states which are orthogonal to $|\psi\rangle$. The maximally polarized state $|\zeta\rangle$ pointing in the $\hat{\mathbf{n}}=(\theta, \phi)$ direction is determined by the equation $\mathbf{F} \cdot \hat{\mathbf{n}}|\zeta\rangle=F|\zeta\rangle$. A convenient (not normalized) representation of this state (which is related to the Schwinger boson representation) is

$$
|\zeta\rangle=\sum_{\alpha=0}^{2 F} \sqrt{\left(\begin{array}{c}
2 F \\
\alpha
\end{array}\right)} \zeta^{\alpha}|F-\alpha\rangle
$$

where $\zeta=e^{i \phi} \tan (\theta / 2)$ is the stereographic mapping of the unit sphere to the complex plane. We define the characteristic polynomial for $|\psi\rangle$ in $\zeta$ to be

$$
f_{\psi}(\zeta) \equiv\langle\psi \mid \zeta\rangle=\sum_{\alpha=0}^{2 F} \sqrt{\left(\begin{array}{c}
2 F \\
\alpha
\end{array}\right)} A_{F-\alpha}^{*} \zeta^{\alpha} .
$$


The values of the $2 F$ complex roots of $f(\zeta),\left\{\zeta_{i}\right\} \equiv$ $\left\{\zeta: f_{\psi}(\zeta)=0\right\}$, (which are in one-to-one correspondence with a set of points on the unit sphere $\left.\left\{\left(\theta_{i}, \phi_{i}\right)\right\}\right)$ determine the coefficients $A_{\alpha}$ and therefore $|\psi\rangle$ up to normalization and an overall phase factor. Most importantly, the symmetries of $|\psi\rangle$ correspond to the operations under which the set of points on the unit sphere $\left\{\left(\theta_{i}, \phi_{i}\right)\right\}$ are invariant. A similar method has been developed in the 19th century mathematics community to solve quintic polynomials in terms of rotations of regular icosahedra [25].

We illustrate our general approach by considering a specific problem of spin-two bosons, first concentrating on the Bose-Einstein condensation (BEC) state in a single optical trap and then extending the discussion to the insulating Mott state in an optical lattice. Spin-two bosons interact with the contact potential

$$
V_{\mathrm{int}}\left(\mathbf{x}_{\mathbf{1}}-\mathbf{x}_{\mathbf{2}}\right)=\delta\left(\mathbf{x}_{\mathbf{1}}-\mathbf{x}_{\mathbf{2}}\right)\left(g_{0} \mathcal{P}_{0}+g_{2} \mathcal{P}_{2}+g_{4} \mathcal{P}_{4}\right)
$$

where $\mathcal{P}_{F}$ projects into the state with total spin $F$ and $g_{F}=$ $2 \pi a_{F} / m$ where $a_{F}$ is the scattering length corresponding to spin $F$. Note the requirement that the wave functions be symmetric under interchange of particles prohibits odd spin projection operators. In the superfluid phase when all atoms occupy the same orbital state, potential (4) leads to the interaction Hamiltonian [15-17] of

$$
\mathcal{H}_{\text {int }}=\frac{1}{2} U_{0} n(n-1)+\frac{1}{2} U_{1} \mathcal{P}_{0}+\frac{1}{2} U_{2}\left(F^{2}-6 n\right),
$$

where $U_{0}=\frac{\gamma}{7}\left(4 a_{2}+3 a_{4}\right), \quad U_{1}=\frac{\gamma}{7}\left(7 a_{0}-10 a_{2}+3 a_{4}\right)$, and $U_{2}=\frac{\gamma}{7}\left(a_{4}-a_{2}\right) \quad(\gamma$ is a positive constant). In Eq. (5) $n=a_{\alpha}^{\dagger} a_{\alpha}$ (here and after sums over repeated indices are implied), where $a_{\alpha}^{\dagger}$ creates a spin-two boson in an eigenstate of $F_{z}$ having eigenvalue $\alpha$. Finally, $F^{2}=$ $\mathbf{F} \cdot \mathbf{F}$, where $\mathbf{F}=a_{\alpha}^{\dagger} \mathbf{T}_{\alpha \beta} a_{\beta}$ and $\mathbf{T}_{\alpha \beta}$ are the spin-two matrices.

The mean-field description for a BEC of $N$ bosons is $\left|\psi_{\mathrm{SF}}\right\rangle=\frac{1}{\sqrt{N !}}\left(A_{\alpha} a_{\alpha}^{\dagger}\right)^{N}|0\rangle$, where the normalized complex coefficients $A_{\alpha}$ are the variational parameters. Since the kinetic energy of the system will not depend on the spin degrees of freedom, it is sufficient to minimize $\left\langle\psi_{\mathrm{SF}}\left|\mathcal{H}_{\text {int }}\right| \psi_{\mathrm{SF}}\right\rangle$ over the variational parameters. To leading order in $N$, we find with this wave function

$$
\begin{aligned}
\left\langle\mathcal{H}_{\text {int }}\right\rangle / N^{2}= & \frac{1}{10} U_{1}\left|2 A_{2} A_{-2}-2 A_{1} A_{-1}+A_{0} A_{0}\right|^{2} \\
& +\frac{1}{2} U_{0}+\frac{1}{2} U_{2}\left(A_{\alpha}^{*} \mathbf{T}_{\alpha \beta} A_{\beta}\right)^{2} .
\end{aligned}
$$

Carrying out the minimization with the constraint of normalization, $A_{\alpha}^{*} A_{\alpha}=1$, we find the phase diagram given in Fig. 1 where the boundaries are given by $a_{2}-a_{4}=$ $\pm \frac{7}{10}\left(a_{0}-a_{4}\right)$ and $a_{2}=a_{4}$. The phases correspond to the coefficients $\left(A_{-2}, A_{-1}, A_{0}, A_{1}, A_{2}\right)$ given by

$$
\begin{aligned}
& \mathcal{F}:(1,0,0,0,0), \mathcal{N}:\left(\frac{\sin (\eta)}{\sqrt{2}}, 0, \cos (\eta), 0, \frac{\sin (\eta)}{\sqrt{2}}\right), \\
& \mathcal{T}:\left(\sqrt{\frac{1}{3}}, 0,0, \sqrt{\frac{2}{3}}, 0\right),
\end{aligned}
$$

which are uniquely determined up to $\mathrm{SO}(3)$ spin rotations by the minimization. Phase $\mathcal{N}$ has the additional degeneracy parameter $\eta$ which does not correspond to a symmetry of the Hamiltonian. One should note, however, that this degeneracy only arises at the mean-field level and will be removed when we include corrections to the mean-field wave function [26]. On the other hand, in real experiments, the quadratic Zeeman effect provides another way of removing this degeneracy which we will discuss. Using the scattering lengths taken from Ref. [15] we also indicate where ${ }^{23} \mathrm{Na}$ and ${ }^{87} \mathrm{Rb}$ will lie in the phase diagram. The same phase boundaries were found by Ciobanu, Yip, and Ho [15] for the superfluid phase. However, we emphasize that there is a continuous degeneracy in the phase $\mathcal{N}$ at the mean-field level.

Symmetries of the phases given by the wave functions in Eq. (7) is not immediate upon first glance. We will now illustrate the utility of our method by classifying the various phases. For spin two, the maximally polarized state as in Eq. (2) is

$$
|\zeta\rangle=\zeta^{4}|-2\rangle+2 \zeta^{3}|-1\rangle+\sqrt{6} \zeta^{2}|0\rangle+2 \zeta|1\rangle+|2\rangle .
$$

Phase $\mathcal{F}$ is clearly the ferromagnetic state. However, it will prove instructive to proceed with our systematic classification scheme. The characteristic polynomial Eq. (3) for this case is simply $f_{\psi_{f}}(\zeta)=\zeta^{4}$ which has the fourfold degenerate root of $\zeta=0$ which corresponds to the north pole of the unit sphere $(\theta, \phi)=(0,0)$. The only symmetries this state possesses are therefore the $\mathrm{U}(1)$ group of continuous rotations about the $z$ axis.

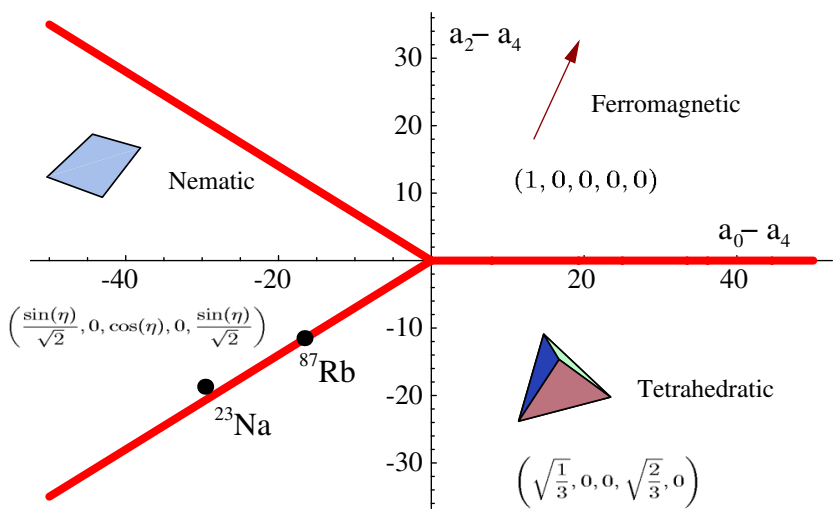

FIG. 1 (color online). Phase diagram for spin-two bosons in a single optical trap. The axes are in units of $a_{B}$. For the Mott insulating state with one boson per site, the phase diagram will be the same with the horizontal and vertical axes corresponding to $\epsilon_{0}-\epsilon_{4}$ and $\epsilon_{2}-\epsilon_{4}$, respectively [see Eq. (12)]. 
We now move on to phase $\mathcal{N}$. Before applying our classification method, it is first useful to consider the order parameters constructed in the conventional way. First, we note that $\langle\mathbf{F}\rangle=0$. Next the eigenvalues of the nematic order parameter Eq. (1) are

$$
\operatorname{Eig}\left(Q_{a b}\right)=\left(\begin{array}{c}
-2 \cos (2 \eta) \\
\cos (2 \eta)+\sqrt{3} \sin (2 \eta) \\
\cos (2 \eta)-\sqrt{3} \sin (2 \eta)
\end{array}\right)
$$

Now, applying our method, the characteristic polynomial is

$$
f_{\psi_{\mathcal{N}}}(\zeta)=\sqrt{\frac{1}{2}} \sin (\eta) \zeta^{4}+\sqrt{6} \cos (\eta) \zeta^{2}+\sqrt{\frac{1}{2}} \sin (\eta) .
$$

The roots of this equation all lie at the vertices of a rectangle. For $0<\eta<\pi / 3, \pi / 3<\eta<2 \pi / 3$, and $2 \pi / 3<\eta<\pi$ the rectangle will lie in the $z y, x y$, and $x z$ planes, respectively. We find that $\eta=n \pi / 3$ (defined $\mathcal{N} 1$ ) corresponds to two sets of double roots at opposite poles. This state will have the transformation properties of a uniaxial nematic. That is, there is the symmetry of continuous rotation about the nematic axis. There are also the additional symmetries of discrete rotations by $\pi$ about any axis in the plane perpendicular to the nematic axis. On the other hand, when $\eta=(n+1 / 2) \pi / 3$ (defined $\mathcal{N} 2)$ the roots lie at the vertices of a square which has no continuous symmetries (contrary to what one might be led to believe from $Q_{a b}$ ). The symmetries here are rotations by $\pi / 2$ about the nematic axis and rotations by $\pi$ about the other two principal axes. For all other values of $\eta$ the nematic will be biaxial, which we refer to as phase $\mathcal{N} 3$. This case has the fewest symmetry operations, the rotations by $\pi$ about the three principal axes.

A route to remove the mean-field degeneracy associated with $\eta$ is to apply an external magnetic field. Since for typical experiments the time it takes a spin state to relax is longer than the trap lifetime [2], it is appropriate to think of $\left\langle F_{z}\right\rangle$ as a conserved quantity, and the linear Zeeman term is unimportant. Thus, the most important effect due to an external magnetic field is due to the quadratic Zeeman term (proportional to $F_{z}^{2}$ ) which can either have a positive or negative coefficient. In the (most common) event that the coefficient is negative, the phase $\mathcal{N} 2$ which transforms as the square will be favored. On the other hand, if the coefficient is positive, the $\mathcal{N} 1$ phase will be favored.

We now consider the remaining region in the phase diagram, phase $\mathcal{T}$, which, as we will see, is tetrahedratically ordered. For this case, $Q_{a b}$ gives no useful information since $\left\langle Q_{a b}\right\rangle=0$ as well as $\langle\mathbf{F}\rangle=0$. Thus, the utility of our classification method will be demonstrated most clearly here. The characteristic polynomial for this situation is $f_{\psi_{\mathcal{T}}}(\zeta)=\sqrt{\frac{1}{3}} \zeta^{4}+2 \sqrt{\frac{2}{3}} \zeta$. The roots of this equation correspond to the points $\left\{\left(\theta_{i}, \phi_{i}\right)\right\}=\{(0,0),(\bar{\theta}, \pi / 3)$, $(\bar{\theta}, \pi),(\bar{\theta}, 5 \pi / 3)\}$, where $\bar{\theta}=2 \tan ^{-1}(\sqrt{2})$. These points lie at the vertices of a regular tetrahedron on the unit sphere. Thus $\left|\psi_{\mathcal{T}}\right\rangle$ has the full symmetry of the point group of the tetrahedron.

We will now consider what happens when the spintwo bosons are in an optical lattice in a Mott insulating state (one per site). Such a system will be described by the Hamiltonian $\mathcal{H}=\mathcal{H}_{\text {kin }}+\mathcal{H}_{\text {Hub }}$ where $\mathcal{H}_{\text {kin }}=$ $-J \sum_{\langle i j\rangle}\left(a_{i \alpha}^{\dagger} a_{j \alpha}+\right.$ H.c. $)$ describes hopping between adjacent sites and $\mathcal{H}_{\text {Hub }}$ gives the on-site interaction

$$
\mathcal{H}_{\mathrm{Hub}, i}=\frac{1}{2} U_{0} n_{i}\left(n_{i}-1\right)+\frac{1}{2} U_{1} \mathcal{P}_{0, i}+\frac{1}{2} U_{2}\left(F_{i}^{2}-6 n_{i}\right) .
$$

Note the similarity between the on-site interaction and Eq. (5). When the sites are completely decoupled, the ground state energy will be independent of the spin state of any given site leading to a macroscopic degeneracy which will be removed by hopping. Treating the hopping perturbatively, we arrive at the effective Hamiltonian

$$
\mathcal{H}_{\text {eff }}=\sum_{\langle i j\rangle}\left[\epsilon_{0} \mathcal{P}_{0}(i j)+\epsilon_{2} \mathcal{P}_{2}(i j)+\epsilon_{4} \mathcal{P}_{4}(i j)\right]
$$

which acts in the subspace of singly occupied sites. Here, $\mathcal{P}_{F}(i j)$ projects neighboring sites at $i j$ into a state with total spin total spin $F$ and $\epsilon_{0}=\frac{-4 J^{2}}{U_{0}+U_{1}-6 U_{2}}, \epsilon_{2}=\frac{-4 J^{2}}{U_{0}-3 U_{2}}$, and $\epsilon_{4}=\frac{-4 J^{2}}{U_{0}+4 U_{2}}$. This effective Hamiltonian is valid in the limit $J \ll U_{0}$. We point out that $\mathcal{H}_{\text {eff }}$ can also be written in terms of powers up to four of spin operators on adjacent sites $\mathbf{S}_{i} \cdot \mathbf{S}_{j}$, but we find the representation in Eq. (12) easier to work with.

When all of the scattering lengths are equal, we will have for the on-site parameters $U_{1}=U_{2}=0$, which will make $\epsilon_{0}=\epsilon_{2}=\epsilon_{4}$. This corresponds to the situation where the Hamiltonian (12) has a special SU(5) symmetry. For this situation, the state with all sites having the same spin wave function $\left|\psi_{\mathrm{MI}}\right\rangle=\prod_{i} A_{\alpha}|\alpha\rangle_{i}$ will be an exact eigenstate of $\mathcal{H}_{\text {eff }}$, which is the ground state. There will still be a rather large degeneracy for this situation since the ground state energy will not depend on the coefficients $A_{\alpha}$. On the other hand, when the scattering lengths begin to differ this degeneracy will be partially lifted. We will now determine the phase diagram using the variational wave function. By evaluating $\left\langle\psi\left|\mathcal{H}_{\text {eff }}\right| \psi\right\rangle$ and minimizing over the set of five complex coefficients $A_{\alpha}$ we find precisely the same coefficients as in (7). Moreover, the phase diagram for this case will be identical with that given in Fig. 1 with the axes changed to $a_{0,2}-a_{4} \rightarrow \epsilon_{0,2}-\epsilon_{4}$.

At the level of virtual hopping Eq. (12) in perturbation theory (second order in $J / U$ ), the states in phase $\mathcal{N}$ are degenerate with respect to the parameter $\eta$. However, this does not correspond to a true symmetry of the parent Hamiltonian. One therefore expects that this degeneracy will be removed at the ring exchange level in perturbation theory (fourth order in $J / U$ ). On the other hand, quantum fluctuations at the virtual hopping level from $\mathcal{H}_{\text {eff }}$ may favor particular phases which can be of the same order as ring exchange. The detailed treatment of this problem is 
(a)

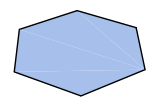

(b)

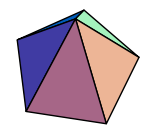

(c)

$$
\left(\frac{1}{\sqrt{2}}, 0,0,0,0,0, \frac{1}{\sqrt{2}}\right)
$$

$(\sin (\eta), 0,0,0,0, \cos (\eta), 0)$

(d)

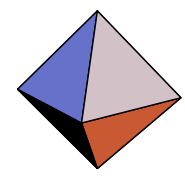

$\left(\frac{\sin (\eta)}{\sqrt{2}}, 0,0, \cos (\eta), 0,0, \frac{\sin (\eta)}{\sqrt{2}}\right)$

FIG. 2 (color online). Some possible phases that can be realized for a spin-three system either in the superfluid or Mott insulating states. Shown are the coefficients of the wave functions $A_{\alpha}$ and the shapes representing the symmetries of the wave functions. The phases transform as the following polyhedra: (a) the hexagon, (b) the pyramid with pentagonal base, (c) the prism, and (d) the octahedron. For phase (c) we have the condition $\tan ^{2}(\eta)<10$. Additional phases similar to the spintwo case (not shown) are the ferromagnetic and nematic states.

beyond the scope of the present work and will be considered elsewhere [26].

Knowing the symmetry properties of the wave function leads to a natural classification of the collective excitations of the system. Breaking continuous symmetries leads to Goldstone bosons; the number of such modes corresponds to the number of broken symmetry generators. Our Hamiltonians will always have an $\mathrm{SO}(3)$ symmetry which corresponds to global spin rotations [at the point where all the scattering lengths are the same there will be a special SU(5) symmetry]. As can be seen in the pictorial representation, the uniaxial nematic phase $\mathcal{N} 1$ has one remaining continuous symmetry and thus break two of the generators of $\mathrm{SO}(3)$. This state will therefore have two Goldstone bosons. The biaxial nematic phases $\mathcal{N} 2$ and $\mathcal{N} 3$, and tetrahedratic phase $\mathcal{T}$ all have no remaining continuous symmetries, so these will have three Goldstone modes. The topological excitations out of the various phases can also be classified using the homotopy theory. The fundamental group describes how different defects combine. For instance, it is known that the fundamental group of the biaxial nematic $\mathcal{N} 3$ is the (nonAbelian) quaternion group [23]. The phase $\mathcal{N} 2$ (transforming as the square) has the larger 16-element dicyclic group [27] for its fundamental group, giving it more types of topological excitations. Finally, the fundamental group of the tetrahedratic state is the binary tetrahedral group with 24 elements [27]. The number of topological excitations $(4,6$, and 6 , respectively) is the number of nontrivial conjugacy classes in each of these groups.

We now will briefly comment on the types of states one can expect for the spin-three problem motivated by recent

interest [19-21]. A similar variational wave function approach for either the superfluid or Mott insulating states as done above can be used for the spin-three case. The characteristic polynomial $f_{\psi}(\zeta)$ will have six roots (instead of four as in the spin-two case). Possible phases that can be realized for such a system are shown in Fig. 2. The boundaries between these phases can be found in [20,21]; the purpose here is to show that our classification scheme can be applied naturally to the spin-three system.

In conclusion, we have presented a classification scheme for cold spinor bosons. We considered spin-two bosons as an illustrative example, which can be realized as a hyperfine state of an alkali atom. In addition to the ferromagnetic and nematic states, we showed that a tetrahedratic ordering can occur for this system. Finally, we discussed an extension to spin-three bosons.

This work was supported by the NSF Grant No. DMR0132874 and the Harvard-MIT CUA. We thank T.-L. Ho, A. Imambekov, A. Kolezhuk, M. Lewenstein, and S. Sachdev for useful discussions.

[1] M. Lewenstein et al., cond-mat/0606771.

[2] J. Stenger et al., Nature (London) 396, 345 (1998).

[3] M.S. Chang et al., Phys. Rev. Lett. 92, 140403 (2004).

[4] M.S. Chang et al., Nature Phys. 1, 111 (2005).

[5] J. Mur-Petit et al., Phys. Rev. A 73, 013629 (2006).

[6] T. L. Ho, Phys. Rev. Lett. 81, 742 (1998).

[7] T. Ohmi and K. Machida, J. Phys. Soc. Jpn. 67, 1822 (1998).

[8] F. Zhou et al., Phys. Rev. B 70, 184434 (2004).

[9] A. Imambekov, M. Lukin, and E. Demler, Phys. Rev. A 68, 063602 (2003).

[10] J. J. Garcia-Ripoll, M. A. Martin-Delgado, and J. I. Cirac, Phys. Rev. Lett. 93, 250405 (2004).

[11] W. X. Zhang, S. Yi, and L. You, Phys. Rev. A 70, 043611 (2004).

[12] J.-S. Bernier, K. Sengupta, and Y. B. Kim, cond-mat/ 0510290 [Phys. Rev. B (to be published)].

[13] A. Widera et al., Phys. Rev. Lett. 95, 190405 (2005).

[14] F. Gerbier et al., Phys. Rev. A 73, 041602 (2006).

[15] C. V. Ciobanu, S. K. Yip, and T. L. Ho, Phys. Rev. A 61, 033607 (2000).

[16] M. Ueda and M. Koashi, Phys. Rev. A 65, 063602 (2002).

[17] L. Zawitkowski et al., cond-mat/0603273.

[18] Fei Zhou and Gordon W. Semenoff, Phys. Rev. Lett. 97, 180411 (2006).

[19] A. Griesmaier et al., Phys. Rev. Lett. 94, 160401 (2005).

[20] L. Santos and T. Pfau, Phys. Rev. Lett. 96, 190404 (2006).

[21] R. Diener and T.-L. Ho, Phys. Rev. Lett. 96, 190405 (2006).

[22] P.B. de Gennes and J. Prost, The Physics of Liquid Crystals (Oxford University, New York, 1995).

[23] N. D. Mermin, Rev. Mod. Phys. 51, 591 (1979).

[24] H. Bacry, J. Math. Phys. (N.Y.) 15, 1686 (1974).

[25] F. Klein, Lectures on the Icosahedron (Dover, New York, 2003).

[26] A. Turner, R. Barnett, and E. Demler (to be published).

[27] H. S. M. Coxeter and W. O. J. Moser, Generators and Relations for Discrete Groups (Springer-Verlag, Berlin,1980), 4th ed. 